\title{
The Nucleolar Protein GLTSCR2 Is an Upstream Negative Regulator of the Oncogenic Nucleophosmin-MYC Axis
}

\author{
Jee-Youn Kim, Young-Eun Cho, and Jae-Hoon Park
}

From the Department of Pathology, College of Medicine, Kyung Hee University, Seoul, Republic of Korea

\author{
Accepted for publication \\ March 19, 2015. \\ Address correspondence to \\ Jae-Hoon Park, M.D., Ph.D., \\ Department of Pathology, \\ College of Medicine, Kyung \\ Hee University, 1 Hoegi- \\ Dong, Dongdemoon-Koo, \\ Seoul 130-701, Republic of \\ Korea. E-mail: jhpark@khu. \\ ac.kr.
}

\begin{abstract}
The transcriptional factor MYC and the nucleophosphoprotein nucleophosmin (NPM) act in concert to regulate the proliferation of both normal and cancer cells. MYC directly interacts with NPM to form an NPM-MYC binary complex, which is recruited to the promoter of MYC target genes to induce the transcription of proteins required for transformation, thus forming an oncogenic NPM-MYC axis. However, the regulatory molecules and mechanisms that control the transcription of MYC target genes by NPM remain to be determined. Herein, we describe a novel function of the nucleolar protein glioblastoma tumorsuppressive candidate region gene 2 (GLTSCR2) in regulating the transcriptional activity of MYC through an NPM-dependent pathway in SK-BR3 breast cancer cells. GLTSCR2 bound to NPM weakly in the nucleolus, but the redistribution of GLTSCR2 to the nucleoplasm increased the binding affinity between the two proteins. Enhancing the GLTSCR2-NPM interaction competitively inhibited the formation of the NPM-MYC binary complex, resulting in a decrease in the recruitment of the NPM-MYC complex to the MYC target gene promoter. This process suppressed the transcriptional and transformational activities of MYC. Thus, our data demonstrated that GLTSCR2 was an upstream negative regulator of the NPM-MYC axis involved in controlling the transcriptional activity of MYC, thereby suggesting that GLTSCR2 may be a novel candidate molecule for suppressing the growth of cancer cells stimulated by MYC hyperactivation. (Am J Pathol 2015, 185: 2061-2068; http://dx.doi.org/10.1016/j.ajpath.2015.03.016)
\end{abstract}

MYC plays an important role in cell growth and tumor development by controlling the transcription of genes involved in proliferation, replication, transformation, and apoptosis. ${ }^{1}$ Thus, tight regulation of MYC activity is critical for normal cell growth and proliferation and for the suppression of cancer development. Consistent with these functions, MYC expression is elevated through transcriptional or post-transcriptional mechanisms in nearly all types of human cancers. ${ }^{2}$ Although the deregulation steps mediating MYC overexpression are rather well defined, the molecular mechanisms through which MYC activates the expression of its target genes, which is essential for MYCinduced transformation and proliferation, are not clear.

Nucleophosmin (NPM)/B23 is a multifunctional nucleolar protein that plays an important role in p53-dependent cell cycle regulation $^{3}$ and tumor development, promoting cell growth and inhibiting apoptosis. ${ }^{4}$ NPM overexpression, which has been observed in gastric, ${ }^{5}$ colonic, ${ }^{6}$ and prostatic carcinomas, ${ }^{7}$ causes enhanced cell growth associated with MYC hyperactivity.
NPM interacts directly with MYC in the nucleoplasm, and the NPM-MYC binary complex binds to the promoter of MYC target genes to induce protein translation, functioning as a key cofactor for MYC-induced hyperproliferation and transformation. ${ }^{8}$ In addition, MYC binds to the NPM promoter, resulting in NPM up-regulation in cells with MYC hyperactivity, ${ }^{9}$ indicating that NPM and MYC act in concert to regulate cell proliferation due to oncogenic stimuli. However, the regulatory mechanisms facilitating the transcription of MYC target genes via the NPM-MYC axis are not clearly understood.

Glioblastoma tumor-suppressive candidate region gene 2 (GLTSCR2/Pict-1) is a nucleolar protein that participates in cell cycle progression and tumor growth by regulating

Supported by National Research Foundation of Korea (NRF) grants 2011-0030072 and NRF-2013R1A2A2A01009006 funded by the Korean government.

Disclosures: None declared. 
Table 1 Primers Used for Real-Time RT-PCR, Promoter Amplification, and ChIP Analysis

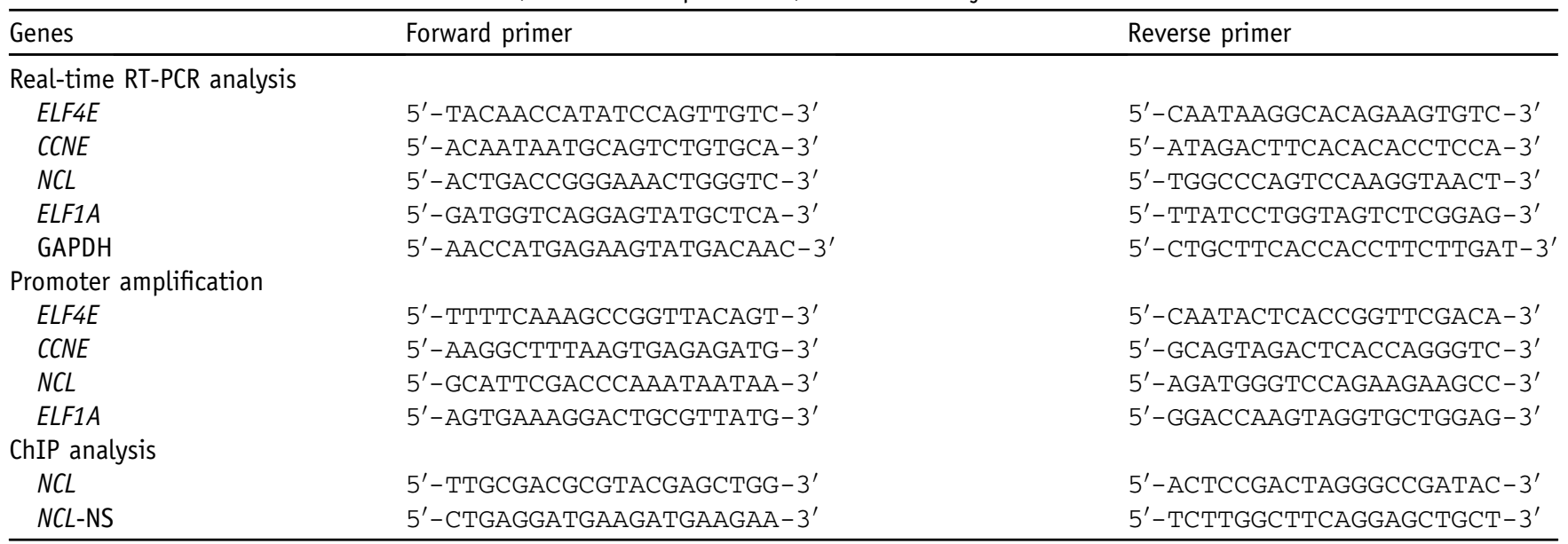

ChIP, chromatin immunoprecipitation; GAPDH, glyceraldehyde-3-phosphate dehydrogenase; nucleolin NS, nucleolin non-specific site.

apoptosis and p53 stability. ${ }^{10-13}$ However, the molecular mechanisms behind these critical cellular processes are largely unknown. Herein, we show that GLTSCR2 functions as an upstream negative regulator of the NPM-MYC axis in cellular transformation by suppressing MYC-induced transforming activity mediated by NPM.

\section{Materials and Methods}

\section{Antibodies, Reagents, and Cell Culture}

SK-BR3 breast cancer cells were obtained from the Korean Cell Line Bank (Seoul, Republic of Korea) and cultured in Dulbecco's modified Eagle's medium supplemented with $10 \%$ fetal bovine serum. SK-BR3/MycER cells were generated by stably infecting cells with retroviruses expressing fusion proteins of MYC and the estrogen receptor. A retrovirus expressing MycER was obtained by transfecting pBabe-MycER and the packaging plasmid pHRCVM8.2 (Addgene, Inc., Cambridge, MA) into $293 \mathrm{~T}$ cells. The anti-GLTSCR2 rabbit polyclonal antibody was purified from antiserum, as described previously. ${ }^{10}$ An anti-NPM mouse monoclonal antibody (Santa Cruz Biotechnology, Inc., Santa Cruz, CA) was used for immunoprecipitation and Western blot analysis. Anti-green fluorescent protein (GFP), anti-V5, anti-fibrillarin, anti-nucleolin, antiupstream binding factor, and matched-isotype control IgG antibodies were purchased from Abcam (Cambridge, UK). Unless otherwise specified, all other reagents were obtained from Sigma-Aldrich, Inc. (St. Louis, MO).

Immunocytochemistry, Immunoprecipitation, Western Blot Analysis, and GST Pull-Down Assay

Western blot analysis, immunostaining, and immunoprecipitation were performed as described previously. ${ }^{10}$ Secondary antibodies recognizing native IgG (Rabbit TrueBlot or Mouse TrueBlot ULTRA; Rockland, Gilbertsville, PA) were used for Western blot analysis after immunoprecipitation. Recombinant
NPM and GLT $\Delta$ NPM-binding domain (NBD) proteins tagged with glutathione $S$-transferase (GST) were produced from bacteria by isopropyl- $\beta$-D-thiogalactopyranoside induction and purified using Glutathione-Sepharose 4B (GE Healthcare, Buckinghamshire, UK), as described previously. ${ }^{11}$ Recombinant GLTSCR2 was purchased from Abnova Corporation (Taoyuan County, Taiwan). For pull-down assays, glutathione GST-NPM or GST proteins were immobilized on GlutathioneSepharose beads in phosphate-buffered saline for 30 minutes at $4^{\circ} \mathrm{C}$. After washing the beads with cold phosphate-buffered saline four times, $100 \mathrm{ng}$ of recombinant GLTSCR2 protein was mixed with the Glutathione-Sepharose beads. Bound proteins were eluted by boiling and detected by Western blot analysis.

\section{Construction of Plasmids and Adenovirus and Knockdown of GLTSCR2}

Plasmids for wild-type and mutant NPM and GLTSCR2 were generated using standard cloning techniques. ${ }^{10}$ Cells were transfected with plasmids using Lipofectamine 2000 (Invitrogen, Carlsbad, CA), according to the manufacturer's recommendations. GLTSCR2 expression was suppressed by infecting cells with lentivirus delivering GLTSCR2-shRNA (Santa Cruz Biotechnology, Inc.).

\section{PLA Data}

A proximity ligation assay (PLA) was performed using a Duolink II detection kit (Olink Bioscience, Uppsala, Sweden), according to the manufacturer's protocols. Briefly, cells were incubated with rabbit anti-MYC and mouse anti-NPM antibodies, and secondary antibodies conjugated with oligonucleotides (PLA probe anti-mouse MINUS and PLA probe anti-rabbit PLUS) were then added. Ligation and amplification were performed using a rolling circle amplification step. The signal was visualized as an individual fluorescent spot. The spots were counted from at least 100 cells. 
A

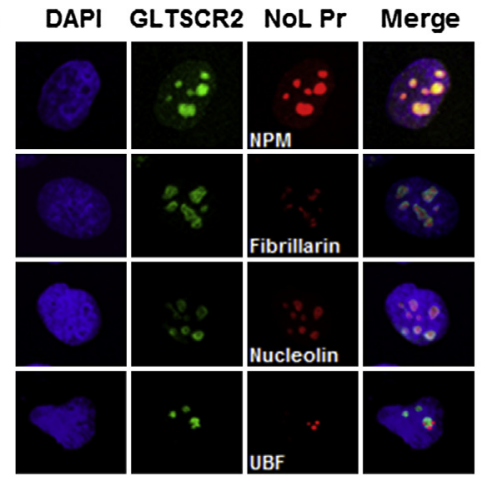

D

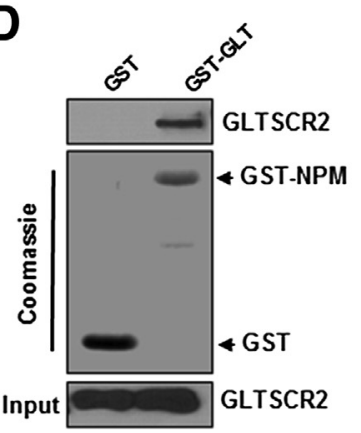

B

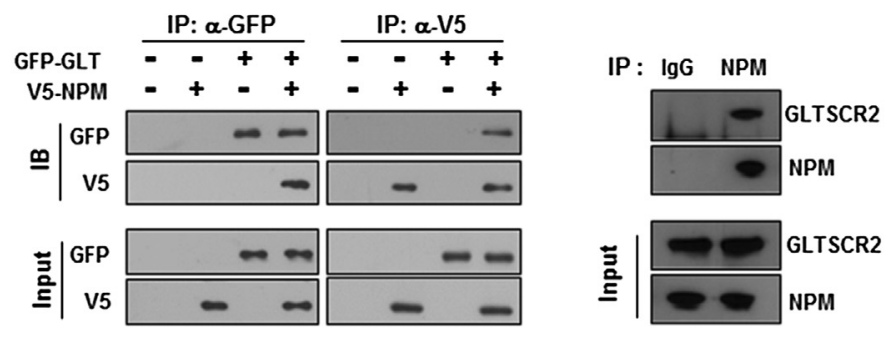

C
E

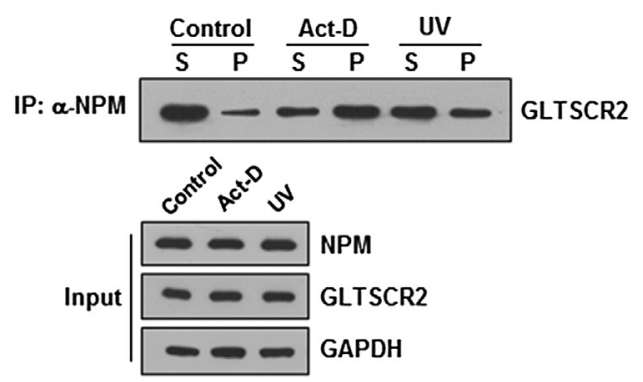

$\mathbf{F}$

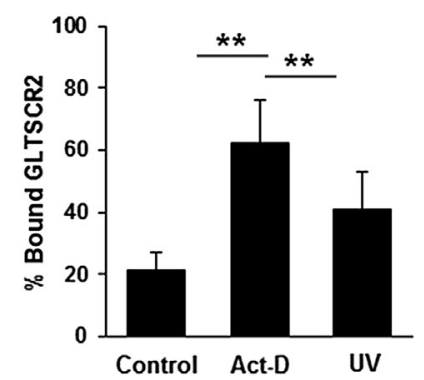

Figure 1 Glioblastoma tumor-suppressive candidate region gene 2 (GLTSCR2) colocalizes and interacts with nucleophosphoprotein nucleophosmin (NPM). A: SK-BR3 cells were co-immunostained with anti-GLTSCR2, anti-NPM, anti-fibrillarin, anti-nucleolin, or anti-upstream binding factor antibodies, and viewed under a confocal microscope. B: SK-BR3 cells were untransfected, singly transfected, or cotransfected with green fluorescent protein (GFP)-tagged GLTSCR2 (GFP-GLT) and V5-tagged NPM (V5-NPM) expression plasmids, as indicated. Cell lysates were immunoprecipitated (IP) using anti-GFP or anti-V5 antibodies, and Western blot analysis was performed using anti-GFP or anti-V5 antibodies and shown in the top panel. A $10 \%$ loading control is shown in the bottom panel. C: Lysates from SK-BR3 cells were immunoprecipitated with anti-NPM antibody or matched isotype IgG, and Western blot analysis was performed using anti-GLTSCR2 or anti-NPM antibody and shown in the top panel. The loading control is shown in the bottom panel. D: A total of $1 \mu \mathrm{g}$ of purified glutathione $S$ transferase (GST) alone or GST-tagged NPM, immobilized on glutathione beads, was incubated with $50 \mathrm{ng}$ of the recombinant GLTSCR2 protein. Bound GLTSCR2 was detected with an anti-GLTSCR2 antibody and shown in the top panel. Recombinant GST and GST-NPM proteins were visualized by Coomassie Blue staining. Western blots for GLTSCR2 are shown in the bottom panel. E: SK-BR3 cells were left untreated (control), treated with $10 \mathrm{ng} / \mathrm{mL}$ actinomycin D (Act-D), or exposed to $20 \mathrm{~J} / \mathrm{m}^{2}$ UV. Cell lysates were immunoprecipitated with excess anti-NPM antibody. The resulting supernatant $(S)$ and precipitate $(P)$ were subjected to Western blot analysis using an anti-GLTSCR2 antibody and shown in the top panel. A representative image is shown. A loading control is shown in the bottom panel. F: Relative amounts of GLTSCR2 were quantified by densitometry, and the ratio of GLTSCR2 in the precipitate/total GLTSCR2 (supernatant plus precipitate) is shown. Data are expressed as the means \pm SD of triplicate determinations (E). ${ }^{* *} P<0.01$. IB, immunoblot; NoL $\operatorname{Pr}$, nucleolar protein.

\section{Real-Time RT-PCR}

mRNA was isolated with Qiazol Lysis Reagent (Qiagen, Valencia, CA). Total RNA $(1 \mu \mathrm{g})$ was reverse transcribed using Moloney Murine Leukemia Virus Reverse Transcriptase (Gibco BRL, Carlsbad, CA) with random hexamer priming. The resulting cDNA was amplified using a LightCycler (Roche Diagnostics Ltd, Lewes, UK). Real-time PCR analysis was performed with LightCycler-FastStart DNA Master SYBR Green I mix (Roche Diagnostics, Basel, Switzerland) and specific primers for elF4E, cyclin $\mathrm{E}$, nucleolin, elF1A, and glyceraldehyde-3-phosphate dehydrogenase (Table 1).

\section{Promoter and ChIP Assays}

elF4E, cyclin E, nucleolin, and elF1A promoter sequences were amplified by PCR from human kidney genomic DNA using upstream and downstream primers (Table 1). Each promoter fragment was cloned in-frame into a pGL2 luciferase reporter vector to generate pGL2-elF4E, pGL2-cyclin E, pGL2-nucleolin, and pGL2-elF1A. Luciferase activity was measured in samples containing equivalent amounts of protein using luminometer and luciferase assay reagents (Promega, Madison, WI). Chromatin immunoprecipitation (ChIP) assays for nucleolin were performed first using anti-MYC antibodies and a ChIP assay kit (Upstate Biotechnology, Lake Placid, $\mathrm{NY}$ ), as recommended by the manufacturer. Chromatin from the first ChIP reaction was eluted with $20 \mathrm{mmol} / \mathrm{L}$ dithiothreitol in Tris-EDTA, according to conventional Re-ChIP protocols. Anti-NPM antibodies were used for the second immunoprecipitation. Immunoprecipitated DNA fragments were analyzed by quantitative PCR using the primer sequences shown in Table 1.

\section{Colony-Forming Assays}

SK-BR3/MycER cells infected with the indicated adenoviruses were plated at a density of $1 \times 10^{4}$ cells/60-mm dish in 
A

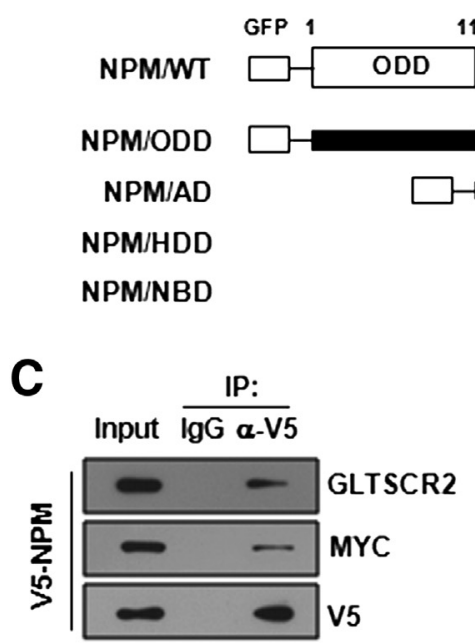

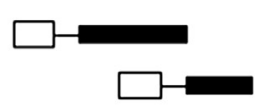
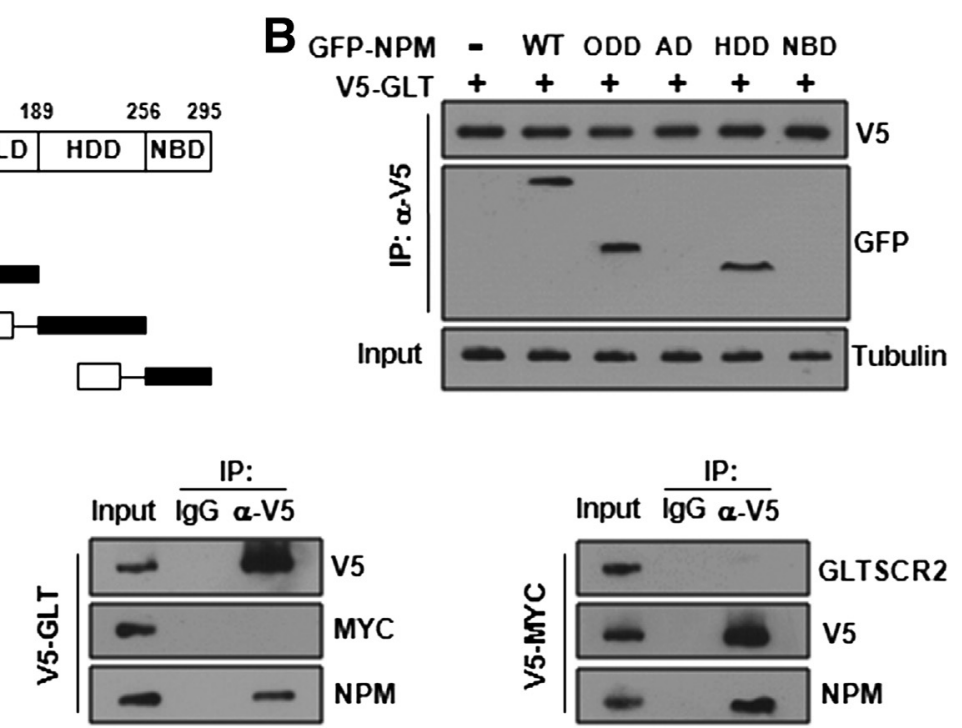

Figure 2 Nucleophosphoprotein nucleophosmin (NPM) forms binary complexes with glioblastoma tumor-suppressive candidate region gene 2 (GLTSCR2) or MYC. A: Schematic diagram of green fluorescent protein (GFP)-tagged NPM and its deletion mutant plasmids. B: Cells were transfected with GFP-tagged wild-type NPM or its deletion mutant, together with a V5-tagged GLTSCR2 (V5-GLT) expression plasmid. Cell lysates were immunoprecipitated (IP) with anti-V5 antibodies, followed by Western blot analysis using the indicated antibodies. A $10 \%$ loading control is shown. C: SK-BR3 cells were transfected with V5-tagged NPM, V5-GLT, or V5-MYC, and cell lysates were immunoprecipitated with an anti-V5 or isotype IgG antibody. Western blot analysis was performed using the indicated antibodies. The $10 \%$ loading control is shown in the left lane of each panel. AD/NLD, acidic domain/nuclear localization domain; HDD, heterodimerization domain; NBD, NPM-binding domain; 0DD, oligodimerization domain; WT, wild type.

soft agar containing Dulbecco's modified Eagle's medium plus $10 \%$ fetal bovine serum. Cells were fed media containing $10 \%$ fetal bovine serum with or without $2 \mu \mathrm{mol} / \mathrm{L} 4$ hydroxytamoxifen (OHT). After incubation at $37^{\circ} \mathrm{C}$ with $5 \%$ $\mathrm{CO}_{2}$ for 3 weeks, cells were stained with $0.005 \%$ crystal violet, and the number and diameter of colonies were measured from triplicate plates. Images were taken with an inverted microscope (model IX71; Olympus Corporation, Tokyo, Japan).

\section{Statistical Analysis}

Statistical analyses were performed using SPSS software, version 12.0 (SPSS, Chicago, IL). Data were analyzed with Fisher exact tests or Pearson $\chi^{2}$ tests. Differences were considered statistically significant when $P<0.05$.

\section{Results}

\section{GLTSCR2 Directly Interacts with NPM}

The nucleolar protein GLTSCR2 completely colocalized with NPM in the nucleolus, but only partially colocalized with other nucleolar proteins, including fibrillarin, nucleolin, and upstream binding factor (Figure 1A). These findings suggest that GLTSCR2 and NPM may interact with each other. To examine whether NPM interacted with GLTSCR2, SK-BR3 cells were cotransfected with GFP-tagged GLTSCR2 (GFP-GLT) and V5-tagged NPM expression plasmids, and co-immunoprecipitation and immunoblotting assays were performed. By using antiGFP antibodies, GFP-GLT was found to associate with V5-tagged NPM (Figure 1B). Conversely, V5-tagged NPM was specifically immunoprecipitated with GFPGLT by anti-V5 antibodies (Figure 1B), indicating that NPM and GLTSCR2 interacted with each other. The interaction between endogenous GLTSCR 2 and NPM was also confirmed (Figure 1C). GST pull-down assays using GST-tagged NPM and recombinant GLTSCR2 indicated that GST-NPM, but not GST alone, directly interacted with GLTSCR2 (Figure 1D).

Although most GLTSCR2 is found in the nucleolus, nucleoplasmic translocation of GLTSCR2 increases the binding affinity between GLTSCR2 and its nucleoplasmic binding partner. ${ }^{11}$ Hence, we next sought to elucidate whether the translocation of GLTSCR2 to the nucleoplasm affected the binding affinity between GLTSCR2 and NPM. Cell lysates from SK-BR3 cells, cultured under physiological conditions or after translocation of GLTSCR2 to the nucleoplasm by treatment with actinomycin $\mathrm{D}$ or exposure to UV radiation, were immunoprecipitated using excess anti-NPM antibody, and both the supernatants and pellets were subjected to Western blot analyses for the detection of GLTSCR2. Enhanced binding of GLTSCR2 to NPM was observed after nucleoplasmic translocation of GLTSCR2 (Figure 1, E and F, and Supplemental Figure S1). Taken together, our results demonstrated that GLTSCR2 interacted directly with NPM and that nucleoplasmic redistribution from the nucleolus increased the binding affinity of the GLTSCR2/NPM interaction. 


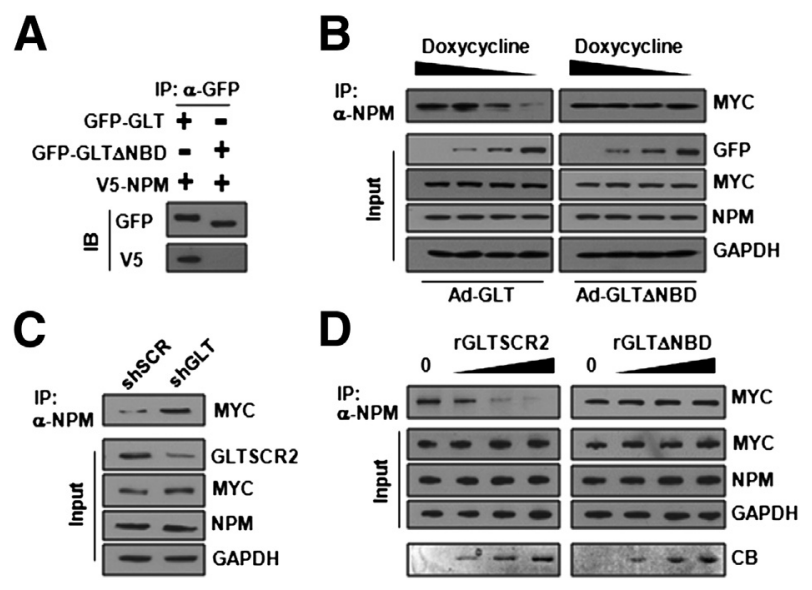

Figure 3 Glioblastoma tumor-suppressive candidate region gene 2 (GLTSCR2) competed with MYC for nucleophosphoprotein nucleophosmin (NPM) binding. A: Cells were cotransfected with V5-tagged NPM and green fluorescent protein (GFP)-GLT or GFP-GLTANPM-binding domain (NBD), and cell lysates were immunoprecipitated (IP) with anti-GFP antibodies, followed by Western blot analysis using anti-GFP or anti-V5 antibodies. B: Cells were infected with adenovirus expressing GFP-tagged GLTSCR2 (AdGLT) or GLT $\triangle$ NBD (Ad-GLT $\triangle N B D$ ) in the presence of varying concentrations of doxycycline $(10,5,2$, or $1 \mathrm{ng} / \mathrm{mL})$. Twenty-four hours after infection, cells were harvested, and lysates were immunoprecipitated with an antiNPM antibody. Western blot analysis was performed with an anti-MYC antibody. Western blots for GFP, MYC, NPM, and glyceraldehyde-3phosphate dehydrogenase (GAPDH) are shown. C: Cell lysates from SKBR3 cells infected with lentivirus expressing scrambled shRNA or shRNA targeted to GLTSCR2 were immunoprecipitated using anti-NPM antibodies, and Western blot analysis was performed using anti-MYC antibodies. A 10\% loading control is shown. D: Increasing amounts $(0,100,250$, and $500 \mathrm{ng})$ of recombinant wild-type GLTSCR2 (rGLTSCR2) or GLTANBD (rGLT $\Delta$ NBD) protein were added to cell lysates, followed by immunoprecipitation with an anti-NPM antibody. Western blot analysis was performed using an antiMYC antibody. Western blots for MYC, NPM, and GAPDH are shown. rGLTSCR2 and rGLTANBD were loaded on a separate gel and visualized by Coomassie Blue (CB) staining. IB, immunoblot; shGLT, short hairpin glioblastoma tumor-suppressive candidate region gene 2; shSCR, short hairpin scrambled RNA.

\section{GLTSCR2 Competes with MYC for Interaction with NPM}

To map the GLTSCR2-binding domain in NPM, a set of deletion mutants of GFP-tagged NPM was generated (Figure 2A). Co-immunoprecipitation assays after cotransfection of each NPM mutant plasmid and V5-tagged GLTSCR2expression plasmid (V5-GLT) revealed that GLTSCR2 bound to both the oligodimerization and heterodimerization domains of NPM (Figure 2B). The heterodimerization domain of NPM is also known to be a binding site for MYC. ${ }^{8}$ Hence, we investigated whether GLTSCR2, NPM, and MYC were present in the form of binary (GLTSCR2-NPM, GLTSCR2-MYC, or NPM-MYC) or trimeric (GLTSCR2-NPM-MYC) complexes in cells. Immunoprecipitation using cell lysates from V5-tagged NPM-, GLT-, or MYC-transfected cells revealed that NPM formed binary complexes with GLTSCR2 or MYC; however, GLTSCR2-MYC binary or NPM-GLTSCR2-MYC trimeric complexes were not formed (Figure 2C). These findings suggested that GLTSCR2 may compete with MYC for NPM binding.
To explore the competitive relationship between GLTSCR2 and MYC for NPM binding, SK-BR3 cells were infected with a doxycycline-inducible (Tetracycline/Off system) adenovirus expressing GFP-tagged GLTSCR2 (Ad-GLT) or GLT $\triangle$ NBD (Ad-GLT $\Delta \mathrm{NBD}$ ) in the presence of decreasing amounts of doxycycline. GLT $\triangle$ NBD is a truncation mutant of GLTSCR 2 lacking an NBD (amino acids 1 to 63 and 346 to 400) (Figure 3A and Supplemental Figure S2). Co-immunoprecipitation was then performed using anti-NPM antibodies. Ectopic expression of GLTSCR2 decreased the interaction between NPM and MYC in a GLTSCR2-dependent manner, whereas GLT $\triangle$ NBD failed to inhibit NPM-MYC binding (Figure 3B). In addition, down-regulation of GLTSCR2 expression by infection with lentivirus expressing GLTSCR2-shRNA increased the MYC-NPM interaction (Figure 3C). To further confirm these findings, we used the PLA, which allows the detection of molecular complexes in situ. ${ }^{14}$ Overexpression or down-regulation of GLTSCR2 suppressed or enhanced the interaction between NPM and MYC, respectively (Supplemental Figure S3).

To elucidate whether the interaction between NPM and MYC was directly inhibited by GLTSCR2, different amounts of recombinant wild-type GLTSCR2 (rGLTSCR2) or mutant GLT $\triangle$ NBD (rGLT $\triangle$ NBD) were added to cell lysates, and Western blot analysis for MYC was performed after immunoprecipitation using anti-NPM antibodies. Consistent with the ectopic overexpression of GLTSCR2 in cells, the interaction between NPM and MYC was suppressed by input of rGLTSCR2 in a concentration-dependent manner, whereas rGLT $\triangle$ NBD did not affect the interaction between NPM and MYC (Figure 3D). Taken together, these results demonstrated that GLTSCR2 competed with MYC for NPM binding.

\section{The Interaction between GLTSCR2 and NPM Suppresses the NPM-Mediated Transcriptional Activity of MYC}

Next, we investigated whether inhibition of the interaction between NPM and MYC, caused by GLTSCR2 overexpression, affected the transcriptional activity of MYC. SK-BR3/MycER cells were infected with Ad-GFP (control), Ad-GLT, or Ad-GLT $\triangle$ NBD, and ectopic GLTSCR2 and GLT $\triangle$ NBD expression levels were regulated by doxycycline. Twelve hours after infection with the adenovirus, cells were treated with OHT, and induction of endogenous MYC target genes, including eIF4E, ${ }^{15}$ cyclin $\mathrm{E},{ }^{16}$ and nucleolin, ${ }^{17}$ was determined. Real-time PCR analyses revealed that activation of MYC by treatment with OHT induced the expression of eIF4E, cyclin E, and nucleolin transcripts in Ad-GFP- or Ad-GLT $\Delta$ NBD-infected cells, whereas ectopic expression of wild-type GLTSCR2 significantly suppressed the induction of MYC target genes in a doxycycline-dependent manner (Figure 4A). The elF1A control gene, which is not regulated by MYC, ${ }^{18}$ did not change after MYC activation (Supplemental Figure S4A). The expression levels of endogenous and ectopic GLTSCR2, MYC, and NPM are shown in Supplemental Figure S4C. Consistent with the 

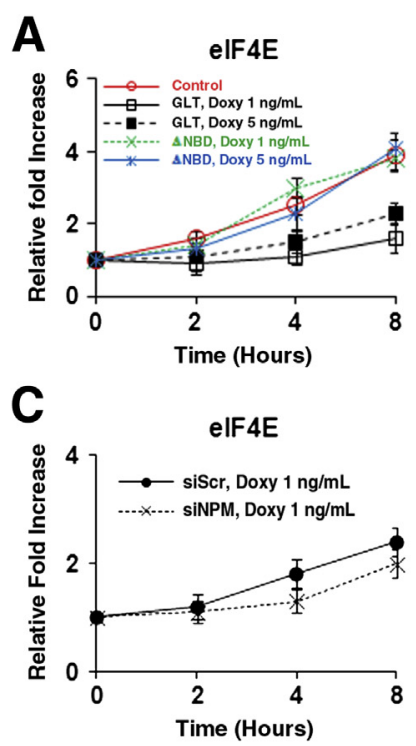
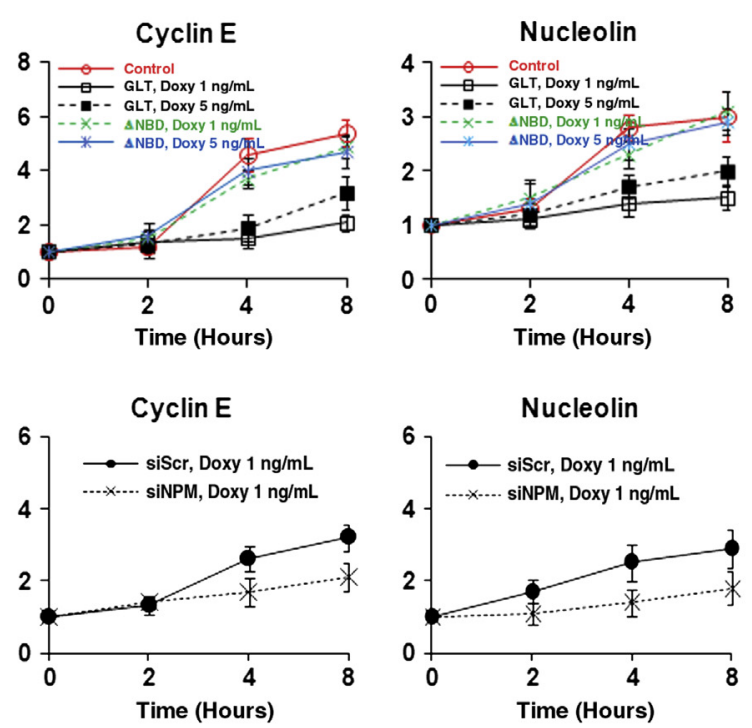

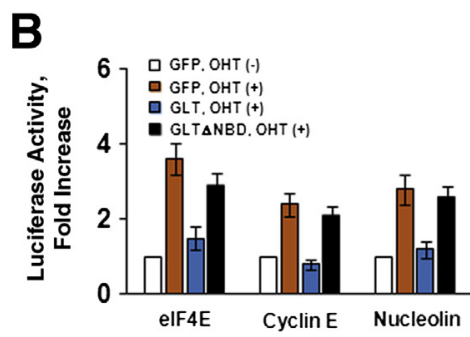

D

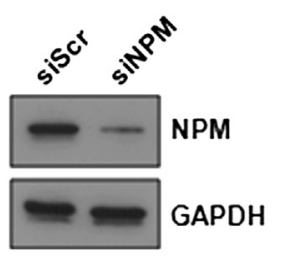

Figure 4 Glioblastoma tumor-suppressive candidate region gene 2 (GLTSCR2) inhibits nucleophosphoprotein nucleophosmin (NPM)-mediated MYC transcriptional activity. A: Real-time RT-PCR analysis of elF4E, cyclin E, and nucleolin mRNAs in SK-BR3/MycER cells infected with adenovirus expressing green fluorescent protein (Ad-GFP; control), Ad-GLT, or Ad-GLTANPM-binding domain (NBD) in the presence of $1 \mathrm{or} 5 \mathrm{ng} / \mathrm{mL}$ doxycycline (Doxy). Cells were infected with each adenovirus before activating MYC for 12 hours with 4-hydroxytamoxifen (OHT). The infection efficiencies were $>95 \%$ in all cases. B: SK-BR3/MycER cells were transiently transfected with $1 \mu \mathrm{g}$ of the indicated luciferase reporter plasmid and co-infected with Ad-GFP, Ad-GLT, or Ad-GLTANBD. At 24 hours after transfection, cells were incubated in medium with or without $2 \mu \mathrm{mol} / \mathrm{L} \mathrm{OHT}$ for 6 hours, and luciferase activities were then determined. The values represent the average luciferase activity of three independent experiments. C: SK-BR3M/MycER cells were transfected with siRNA targeted to GLTSCR2 (siNPM) or scrambled siRNA (siScr) for 3 days, and then the transcriptional activities of MYC in relation with elF4E, cyclin E, and nucleolin were determined by real-time RT-PCR. D: Lysates from SK-BR3M/MycER cells transfected with siNPM or siScr were subjected to Western blot analysis using the indicated antibodies. Data are given as means \pm SD (B). GAPDH, glyceraldehyde-3-phosphate dehydrogenase.

results of real-time PCR, GLTSCR2 expression suppressed the promoter activities of eIF4E, cyclin E, and nucleolin after activation of MYC, whereas GFP or GLT $\triangle$ NBD expression failed to inhibit the activities of these promoters (Figure 4B). The elF1A promoter was not affected by GLTSCR 2 expression (Supplemental Figure S4B).

Next, we determined the effects of NPM down-regulation on the transcriptional activity of MYC to further support our observation that GLTSCR2 regulated the interaction between NPM and MYC. SK-BR3/MycER cells were transfected with siRNA targeting NPM or scrambled siRNA, and the transcriptional activity of MYC on target genes was then determined. The transcriptional activity of MYC was reduced by NPM down-regulation, indicating that the transcriptional activity of MYC was determined by the expression levels of GLTSCR2, NPM, and MYC (Figure 4C). The expression level of NPM in SK-BR3/MycER cells transfected with siRNA targeting NPM or scrambled siRNA is shown in Figure 4D.

Next, we determined whether GLTSCR2 affected the recruitment of the NPM-MYC complex at the nucleolin promoter in SK-BR3 cells. Sequential ChIP assays revealed that recruitment of the NPM-MYC complex to the nucleolin promoter was reduced in Ad-GLT-infected cells, but not in Ad-GFP- or Ad-GLT $\Delta$ NBD-infected cells (Figure 5). Taken together, these results indicated that the association between GLTSCR2 and NPM inhibited the NPM-mediated induction of MYC target genes.
GLTSCR2 Suppresses the NPM-Mediated Transforming Activity of MYC

To explore the effects of GLTSCR2 overexpression on MYCinduced transforming activity mediated by NPM, SK-BR3/ MycER cells were infected with Ad-GLT or Ad-GLT $\triangle N B D$, and anchorage-independent growth assays were performed. The expression of ectopic MYC, induced by treatment with

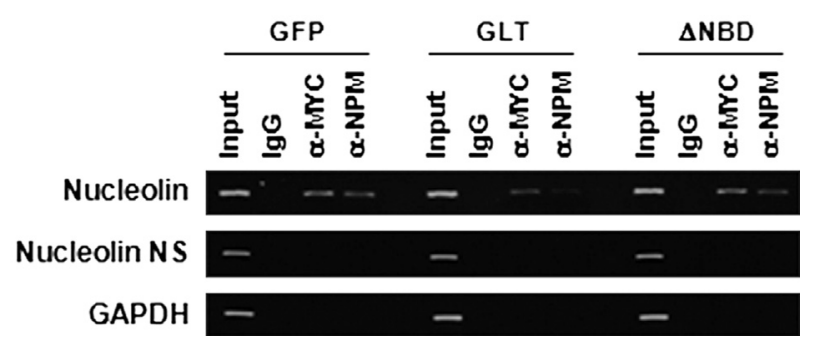

Figure 5 Glioblastoma tumor-suppressive candidate region gene 2 (GLTSCR2) affects the recruitment of the nucleophosphoprotein nucleophosmin (NPM)-MYC complex at the nucleolin promoter. SK-BR3 cells were infected with adenovirus expressing green fluorescent protein (Ad-GFP), Ad-GLT, or AdGLTSNPM-binding domain (NBD) for 24 hours, treated with formaldehyde, and then sonicated. The cross-linked DNA-protein complex was immunoprecipitated with an anti-MYC or isotype IgG antibody. Eluted chromatin from the first chromatin immunoprecipitation (ChIP) reaction was immunoprecipitated with anti-NPM antibodies, followed by PCR amplification of the nucleolin promoter. Immunoprecipitates by IgG served as negative controls. Input chromatin (Input) represents a portion of the sonicated chromatin before immunoprecipitation. PCR for the nucleolin non-specific site (Nucleolin NS) and glyceraldehyde-3-phosphate dehydrogenase (GAPDH) promoter was performed as a negative control. 

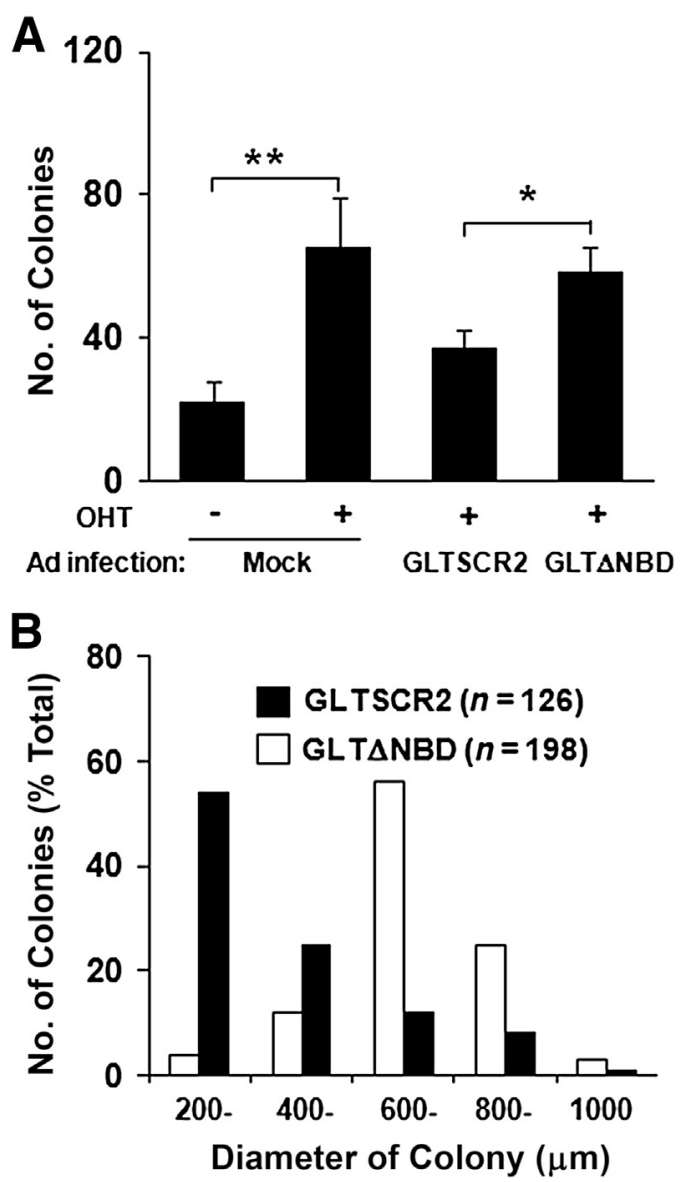

Figure 6 Glioblastoma tumor-suppressive candidate region gene 2 (GLTSCR2) suppresses the transforming activity of MYC. A: SK-BR3/MycER cells were infected with adenovirus expressing green fluorescent protein-tagged GLTSCR2 (Ad-GLT) or Ad-GLTAnucleophosmin-binding domain (NBD) and plated at $10^{4}$ cells/60-mm dish in soft agar. Cells were fed fresh media containing $10 \%$ fetal bovine serum and $2 \mu \mathrm{mol} / \mathrm{L}$ 4-hydroxytamoxifen (OHT) every 3 days. Three weeks after seeding, colony formation was assessed as described in Materials and Methods. B: The distribution of the diameters of colonies from Ad-GLT-infected cells and Ad-GLTANBD-infected cells is shown as a histogram. Data are shown as the means \pm SD number of colonies from three independent experiments (A). $n=126$ (B, Ad-GLT-infected cells); $n=198$ (B, Ad-GLT $\triangle$ NBD-infected cells). ${ }^{*} P<0.05,{ }^{* *} P<0.01$.

OHT, increased colony formation compared with that in untreated cells. Interestingly, GLTSCR2 overexpression significantly suppressed the anchorage-independent growth of cells exhibiting MycER activation, whereas GLT $\Delta$ NBD overexpression did not suppress the colony formation of SK-BR3/ MycER cells (Figure 6A). In addition, SK-BR3/MycER cells infected with Ad-GLT formed smaller colonies (average diameter, $224 \mu \mathrm{m}$ ) compared with cells that were infected with GLT $\Delta$ NBD (average diameter, $695 \mu \mathrm{m}$ ) (Figure 6B). These results indicated that GLTSCR2 inhibited the cellular transformation induced by NPM-mediated MYC activation.

\section{Discussion}

The nucleolar protein NPM is a key protein responsible for both oncogenic and tumor-suppressive activities. In response to oncogenic stresses, redistributed NPM stabilizes p53 by inactivating murine double minute 2 through an alternative reading frame-dependent pathway. ${ }^{19}$ On the other hand, NPM is linked to the growth and proliferation of normal and cancer cells through MYC-dependent pathways. The NPM-MYC complex, which is formed by direct interaction between NPM and MYC, is recruited to and activates the promoter of MYC target genes required for cell growth and transformation, such as elF4E and cyclin-dependent kinase $4 .{ }^{8}$ In addition, redistribution of NPM by oncogenic stress increases the binding between NPM and MYC, potentially promoting further cell growth through MYC pathways, which may result in enhanced growth of tumor cells. ${ }^{8}$ However, the dramatic effects of NPM on MYC were not due to a significant increase in total NPM expression, ${ }^{20}$ suggesting the presence of additional unknown mechanisms through which NPM regulates MYC transcriptional activity.

The immunoprecipitation and PLA assays indicated that regulation of the NPM-MYC axis by GLTSCR2 resulted from competitively suppressing NPM-MYC complex formation in the nucleoplasm. Our findings were as follows: i) GLTSCR2 and MYC shared a common NPM binding domain, ii) the GLTSCR2-NPM-MYC tertiary complex was not detected by conventional and sequential immunoprecipitation (data not shown), and iii) the GLT $\triangle$ NBD mutant, which did not bind NPM, failed to suppress NPM-MYC complex formation. Furthermore, our results using recombinant proteins suggested that the suppression of the interaction between NPM and MYC by GLTSCR2 could not be attributed to transcriptional mechanisms. However, most GLTSCR2 resides in the nucleolus, ${ }^{21}$ whereas most MYC is localized in the nucleoplasm. Thus, the mechanisms through which GLTSCR2 effectively regulates the NPM-MYC axis are unknown. Our findings demonstrated that nucleoplasmic redistribution of GLTSCR2, as well as GLTSCR2 overexpression, played important roles in the regulation of the NPM-MYC axis, possibly by increasing the association between GLTSCR2 and NPM. Because nucleoplasmic redistribution of GLTSCR2 occurs under DNA damage conditions or nucleolar stresses, including hypoxia and serum deprivation, ${ }^{22}$ GLTSCR2 may be a candidate molecule controlling the NPM-MYC axis to inhibit cell growth or proliferation under unfavorable conditions.

GLTSCR2 acts as both a tumor suppressor and an oncogenic protein, similar to NPM. ${ }^{13,23}$ Studies on the oncogenic activity of GLTSCR2 have demonstrated that once GLTSCR2 is depleted in the nucleolus, it is not able to seize nucleolar RPL11. As a result, RPL11 is released into the nucleoplasm, where it suppresses murine double minute $2-$ mediated p53 degradation. ${ }^{13}$ In contrast, our work indicated that the tumorsuppressive activity of GLTSCR2 in the regulation of the NPM-MYC axis resulted from the translocation of nucleolar GLTSCR2 to the nucleoplasm as well as GLTSCR2 expression levels. Similarly, NPM in the nucleolus potentiates cell growth and proliferation through ribosomal biogenesis and rRNA maturation, ${ }^{24}$ whereas NPM released from the nucleolus as a result of ribosomal stress stabilizes p53 in association with alternative reading frame. ${ }^{25}$ 
In summary, our current data showed that GLTSCR2 released from the nucleolus interacted with NPM and competitively inhibited the formation of the NPM-MYC complex, thereby suppressing MYC-induced hyperproliferation and transformation controlled by NPM. Our study demonstrated that GLTSCR2 was an upstream negative regulator of the NPM-MYC axis. Therefore, regulating the oncogenic activity of MYC by GLTSCR2 may be a novel therapy for suppressing the growth of cancer cells caused by hyperactivation of MYC.

\section{Supplemental Data}

Supplemental material for this article can be found at http://dx.doi.org/10.1016/j.ajpath.2015.03.016.

\section{References}

1. Dang CV: c-Myc target genes involved in cell growth, apoptosis, and metabolism. Mol Cell Biol 1999, 19:1-11

2. Junttila MR, Westermarck J: Mechanisms of MYC stabilization in human malignancies. Cell Cycle 2008, 7:592-596

3. Huang N, Negi S, Szebeni A, Olson MO: Protein NPM3 interacts with the multifunctional nucleolar protein B23/nucleophosmin and inhibits ribosome biogenesis. J Biol Chem 2005, 280:5496-5502

4. Feuerstein N, Spiegel S, Mond JJ: The nuclear matrix protein, numatrin (B23), is associated with growth factor induced mitogenesis in Swiss 3T3 fibroblasts and with $\mathrm{T}$ lymphocyte proliferation stimulated by lectins and anti-T cell antigen receptor antibody. J Cell Biol 1988, 107:1629-1642

5. Tanaka M, Sasaki H, Kino I, Sugimura T, Terada M: Genes preferentially expressed in embryo stomach are predominantly expressed in gastric cancer. Cancer Res 1992, 52:3372-3377

6. Nozawa Y, Van Belzen N, Van der Made AC, Dinjens WN, Bosman FT: Expression of nucleophosmin/B23 in normal and neoplastic colorectal mucosa. J Pathol 1996, 178:48-52

7. Subong EN, Shue MJ, Epstein JI, Briggman JV, Chan PK, Partin AW Monoclonal antibody to prostate cancer nuclear matrix protein (PRO: 4-216) recognizes nucleophosmin/B23. Prostate 1999, 39:298-304

8. Li Z, Boone D, Hann SR: Nucleophosmin interacts directly with c-Myc and controls c-Myc-induced hyperproliferation and transformation. Proc Natl Acad Sci U S A 2008, 105:18794-18799

9. Zeller KI, Haggerty TJ, Barrett JF, Guo Q, Wonsey DR, Dang CV: Characterization of nucleophosmin (B23) as a Myc target by scanning chromatin immunoprecipitation. J Biol Chem 2001, 276:48285-48291

10. Yim JH, Kim YJ, Ko JH, Cho YE, Kim SM, Kim JY, Lee S, Park JH: The putative tumor suppressor gene GLTSCR2 induces PTENmodulated cell death. Cell Death Differ 2007, 14:1872-1879
11. Lee S, Kim JY, Kim YJ, Seok KO, Kim JH, Chang YJ, Kang HY, Park JH: Nucleolar protein GLTSCR2 stabilizes p53 in response to ribosomal stresses. Cell Death Differ 2012, 19:1613-1622

12. Okahara F, Itoh K, Nakagawara A, Murakami M, Kanaho Y, Maehama T: Critical role of PICT-1, a tumor suppressor candidate, in phosphatidylinositol 3,4,5-trisphosphate signals and tumorigenic transformation. Mol Biol Cell 2006, 17:4888-4895

13. Sasaki M, Kawahara K, Nishio M, Mimori K, Kogo R, Hamada K, Itoh B, Wang J, Komatsu Y, Yang YR, Hikasa H, Horie Y, Yamashita T, Kamijo T, Zhang Y, Zhu Y, Prives C, Nakano T, Mak TW, Sasaki T, Maehama T, Mori M, Suzuki A: Regulation of the MDM2-P53 pathway and tumor growth by PICT1 via nucleolar RPL11. Nat Med 2011, 17:944-951

14. Soderberg O, Gullberg M, Jarvius M, Ridderstrale K, Leuchowius KJ, Jarvius J, Wester K, Hydbring P, Bahram F, Larsson LG, Landegren U: Direct observation of individual endogenous protein complexes in situ by proximity ligation. Nat Methods 2006, 3:995-1000

15. Jones RM, Branda J, Johnston KA, Polymenis M, Gadd M, Rustgi A, Callanan L, Schmidt EV: An essential E box in the promoter of the gene encoding the mRNA cap-binding protein (eukaryotic initiation factor 4E) is a target for activation by c-myc. Mol Cell Biol 1996, 16:4754-4764

16. Leone G, DeGregori J, Sears R, Jakoi L, Nevins JR: Myc and Ras collaborate in inducing accumulation of active cyclin E/Cdk2 and E2F. Nature 1997, 387:422-426

17. Greasley PJ, Bonnard C, Amati B: Myc induces the nucleolin and BN51 genes: possible implications in ribosome biogenesis. Nucleic Acids Res 2000, 28:446-453

18. Knoepfler PS, Zhang XY, Cheng PF, Gafken PR, McMahon SB, Eisenman RN: Myc influences global chromatin structure. EMBO J 2006, 25:2723-2734

19. Kurki S, Peltonen K, Latonen L, Kiviharju TM, Ojala PM, Meek D, Laiho M: Nucleolar protein NPM interacts with HDM2 and protects tumor suppressor protein p53 from HDM2-mediated degradation. Cancer Cell 2004, 5:465-475

20. Li Z, Hann SR: The Myc-nucleophosmin-ARF network: a complex web unveiled. Cell Cycle 2009, 8:2703-2707

21. Kalt I, Levy A, Borodianskiy-Shteinberg T, Sarid R: Nucleolar localization of GLTSCR2/PICT-1 is mediated by multiple unique nucleolar localization sequences. PLoS One 2012, 7:e30825

22. Kim JY, Seok KO, Kim YJ, Bae WK, Lee S, Park JH: Involvement of GLTSCR2 in the DNA damage response. Am J Pathol 2011, 179: 1257-1264

23. Kim YJ, Cho YE, Kim YW, Kim JY, Lee S, Park JH: Suppression of putative tumour suppressor gene GLTSCR2 expression in human glioblastomas. J Pathol 2008, 216:218-224

24. Itahana K, Bhat KP, Jin A, Itahana Y, Hawke D, Kobayashi R, Zhang Y: Tumor suppressor ARF degrades B23, a nucleolar protein involved in ribosome biogenesis and cell proliferation. Mol Cell 2003, 12:1151-1164

25. Lee C, Smith BA, Bandyopadhyay K, Gjerset RA: DNA damage disrupts the p14ARF-B23(nucleophosmin) interaction and triggers a transient subnuclear redistribution of p14ARF. Cancer Res 2005, 65:9834-9842 